\title{
Visible Light Positioning using Bayesian Filters
}

\author{
Robin Amsters ${ }^{(0)}$, Dimiter Holm, Joren Joly, Eric Demeester $\left.{ }^{(}\right)$, Nobby Stevens ${ }^{(}$, and Peter Slaets $\left.{ }^{(}\right)$
}

\begin{abstract}
Visible light positioning has the potential to be a cost-effective technology for accurate indoor positioning. However, existing approaches often require large amounts of incoming data, usually in the form of high resolution images or dense lighting distributions. Additionally, a line of sight between transmitter and receiver is generally required at all times. In this work, we present a positioning approach that combines measurements from a camera, encoders and a gyroscope. We compare multiple algorithms for fusing these data, namely an extended Kalman filter, a particle filter and a hybrid approach. The end result is a system that provides location estimates even with sparse lighting distributions and temporary outages, yet achieves an average accuracy of 2 to $4 \mathrm{~cm}$. Even in the 95th percentile of the cumulative error distribution, accuracy can be as low as 2 $\mathrm{cm}$ and is often lower than $10 \mathrm{~cm}$. Moreover, due to the use of a low-resolution camera (640x480 pixels) and efficient fusion algorithms, the latency is relatively low on a standard laptop (between 5.6 and 21 milliseconds). Even on a low-cost embedded board, latency generally does not exceed 100 milliseconds. We validate our approach experimentally and show that it is robust under a wide range of illumination conditions.
\end{abstract}

Index Terms-Indoor Positioning, Visible Light Positioning, Sensor Fusion, Mobile Robots, Kalman Filtering, Particle Filter

\section{INTRODUCTION}

$\mathbf{L}$ OCATION information is a prerequisite for many autonomous systems. Cars, drones, robots, etc. all require positioning data in order to reach their target locations. Outdoors, Global Navigation Satellite Systems (GNSS) are often used for this purpose. A constellation of artificial satellites transmits location and timing data from outer space to a receiver on earth. One of the most well known examples of a GNSS is the global positioning system (GPS). Indoors, however, such systems often perform poorly, as the signals are challenging to receive through walls. Moreover, the accuracy is often not sufficient for indoor applications [1]. Therefore, Indoor Positioning Systems (IPS) have been proposed to provide indoor location data. Examples of IPS include Wireless Local Area Networks (WLAN), Bluetooth and Ultra-Wideband radio (UWB). WLAN network infrastructure is already present in many indoor environments. As a result, WLAN positioning can simply be implemented in software. The downside is its lack of accuracy, which is generally a few meters and increases for moving targets [2]. Bluetooth is better suited for proximity

R. Amsters, E. Demeester and P. Slaets are with the Department of Mechanical Engineering, KU Leuven, Leuven, Belgium (email: robin.amsters@kuleuven.be; $\quad$ eric.demeester@kuleuven.be; peter.slaets@kuleuven.be)

Robin Amsters is an SB fellow of the Research Foundation Flanders (FWO) under grant agreement 1 S57720N.

N. Stevens is with the Department of Electrical Engineering, KU Leuven, Leuven, Belgium (e-mail: nobby.stevens@kuleuven.be).

Manuscript received February 11, 2020; revised June 8, 2020 detection than location tracking. Nevertheless, the technology is quite popular due to its low cost. Using Bluetooth Low Energy (BLE), beacons broadcast signals that can be received by smartphones or special tags. Accuracies as low as $1 \mathrm{~m}$ can be reached [3]. UWB radios have a bandwidth of more than $500 \mathrm{MHz}$. Such a large bandwidth results in very short pulses in the time domain, which in turn makes it easier to determine the time of arrival [4]. As such, the accuracy of UWB is higher than the previously discussed technologies due to the decreased influence of reflections ( $\mathrm{cm}$ to $\mathrm{dm}$ range [5]). Nevertheless, UWB systems are significantly more expensive than other radio-frequency based solutions.

As solid state lighting becomes more widespread, a new type of wireless communication based on optical signals has become viable. In Visible Light Communication (VLC), Light Emitting Diodes (LED) are switched at frequencies imperceptible to the human eye. A receiver can decode the signal, thus establishing a wireless transmission link [6]. IPS that use visible light signals are also referred to as Visible Light Positioning (VLP) systems. Generally, transmitters in a VLP system emit some sort of identifier (ID), such as a unique code or frequency [7]. The coordinates corresponding to each light source can be emitted as part of the signal, or can be stored at the receiver side with a link to the identifier [8] (for example, a modulation frequency of $2 \mathrm{kHz}$ could correspond to a light source at the beginning of a particular hallway). VLP systems can often reuse existing lighting infrastructure, thereby lowering the investment and invasiveness of such systems. Additionally, accuracy can be in the decimeter to centimeter range [8]. Both photodiodes (PD) and cameras can be used as VLP receivers. Photodiodes are very low-cost, easily integrated in a variety of devices and can be used for high accuracy positioning [8]. However, additional hardware is needed to condition the signal and sample it at high frequencies. Moreover, the data is spatially sparse compared to image sensors. On the other hand, images generally require more computational resources to process than photocurrents. Therefore, image-based VLP systems usually have higher latencies. Regardless of the receiver, many implementations need multiple LEDs to be in range, in order to perform trilateration or triangulation [8]. Such approaches rely on dense deployments of beacons, thereby increasing the overall system cost. Some proposed methods require only one transmitter to be in view. For example, in [9], the authors proposed proximity-based positioning for VLP, which means that the receiver position was set to the position of the closest detected light fixture. Such a scheme reduces complexity, as one only needs to be able to decode the identifiers. However, 
accuracy is defined by the density of the lighting installation. [10] also proposed a single LED positioning system. Rather than treating the transmitter as a point, the authors used the geometric properties of the circular light to recover position and orientation. On average, the accuracy was between 13 $\mathrm{cm}$ and $17.5 \mathrm{~cm}$, depending on ceiling height. This method does require additional markers to be placed on the armature. Moreover, a very high resolution camera was used (7712 by 4352 pixels), which significantly increased the computational complexity and system cost. The system described in [11] used the projection of a circular light in a similar way, yet does not require additional markers. Instead, data from the Inertial Measurement Unit (IMU) of a smartphone was used. With this method, the authors were able to achieve an average accuracy of $11.2 \mathrm{~cm}$. In the case of a Non-direct Line Of Sight (NLOS) to a light source, both works are unable to determine the receiver location.

Our proposed approach uses sensor fusion, which means that the information from multiple sensors is combined into a single estimate of the state of a system. In indoor positioning, sensor fusion is receiving more attention, as it can improve both robustness and accuracy [12]. As VLP is a relatively novel technology, fusion with complementary sensors has not been investigated extensively. Literature on the subject is often based exclusively on simulations [13], [14], yet some examples of experimental evaluations are available. For example, the Lookup system proposed in [15] used a camera with a fisheye lens for VLP beacon detection, and combined these data with an accelerometer and magnetometer. However, the approach only worked for static positioning, as the ID transmission and detection times were relatively long $(1.7 \mathrm{~s}$ and $4 \mathrm{~s}$, respectively) due to the long bit transmission time. Accuracy was on the order of a few decimeters, when 3 or 4 beacons were visible. [16] utilized an Extended Kalman Filter (EKF) to fuse VLP data from a photodiode with an Inertial Measurement Unit (IMU). The mean accuracy was $14.5 \mathrm{~cm}$, yet 3 lights needed to be visible in order to estimate a position, otherwise accuracy quickly degraded. The follow up work [17] evaluated the Particle Filter (PF) as a fusion algorithm. Results were similar to the EKF, yet were slightly more accurate. Computational requirements or latency were not mentioned explicitly. The VLIP system in [18] used an accelerometer to cover VLP outages, and graph optimization to fuse the sensor data. Experimental evaluation was performed with Augmented Reality (AR) tags, rather then LED beacons. Four tags needed to be visible at all times in order to use the VLP data. Finally, in [19], the authors described another experimentally validated VLP system. A PF was used to track the location of a camera. The approach could use one or more transmitters, was robust to partial occlusions and fluorescent lighting and had a high update rate $(+-50 \mathrm{~Hz})$. Additionally, the average accuracy was relatively high at $2.95 \mathrm{~cm}$. However, experiments were performed in a relatively small testing area ( 2.1 by 1.1 meters). Additionally, the particle weights were updated by matching image sub-regions to a template. This implies that all LEDs must have the same (known) shape.

The EKF and the PF are two of the most used sensor fusion algorithms. Both have unique advantages that make them suited for different applications. The EKF is highly efficient, and performs well in the presence of moderate non-linearities. Contrary to the PF, the EKF does require an initial position estimate, and is generally unable to cope with multi-modal estimates. Additionally, the PF performs better in the presence of high non-linearities, yet requires significantly more computational power. In this paper, we therefore propose a combination of these approaches, in order to leverage the advantages of both yet minimize their drawbacks. A relatively large number of possible hybrid filters exist, and have been proposed for a wide range of applications. In the field of positioning, [20] compared Kalman filter based scan matching to grid-based Markov localization. The authors concluded that the Kalman filter is more accurate when sufficient information is available, yet that Markov localization is more robust. Their findings suggest that a combination of these techniques would be favorable. This combination was evaluated in [21], and compared to other methods in [22]. Results showed that the combined filter outperformed both of its components, and inherited the accuracy of Kalman filtering and the robustness and re-localization speed of the Markov method. Additionally, the Markov/Kalman filter performed better than other hybrid filters. Literature that compared hybrid filters to their components generally reported similar performance gains [23], [24]. A comprehensive overview of hybrid particle/Kalman filters can be found in [25]. To the best of our knowledge, this idea has not been applied to VLP.

In this work, we outline a system that fuses VLP camera data with proprioceptive sensors commonly found on a mobile robot. We perform an experimental evaluation under a range of lighting conditions. Position estimation is performed with both an EKF and a PF. Additionally, we propose a hybrid particle/Kalman $(\mathrm{PaKa})$ filter that combines the advantages of both approaches. In summary, our main contributions are:

- We make use of a low-resolution camera (640 by 480 pixels) and fuse the data with odometry sensors. This leads to very fast processing times.

- The proposed approach works with sparse lighting distributions. Therefore, there is no need for a constant LOS component to multiple LEDs.

- Even with the reduced processing time, high accuracy is maintained.

- The system is tested and proven effective under a range of conditions.

- The hybrid filter is shown to have an accuracy better than both individual filters, whilst only minimally reducing the update rate.

The rest of this paper is structured as follows; Section II elaborates the materials and methods used in this work. Experimental results are presented in Section III and discussed in Section IV. Finally, a conclusion is drawn in Section V.

\section{MATERIALS AND METHODS}

\section{A. Experimental setup}

Experiments were performed in a lab environment of approximately $2 \mathrm{~m}$ by $2 \mathrm{~m}$, with 4 transmitters mounted at a 


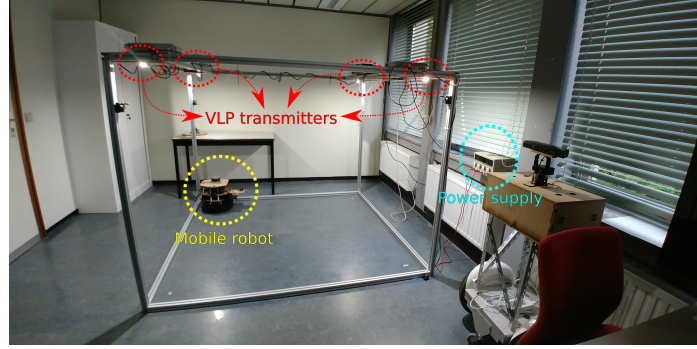

Fig. 1: Experimental setup.

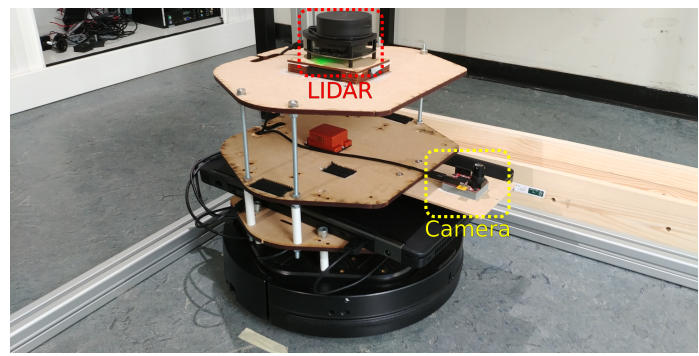

Fig. 2: Mobile robot with sensor platform.

height of approximately $1.5 \mathrm{~m}$ (see Fig. 1). The LEDs were powered by a lab bench power supply, and were switched by a MOSFET connected to a signal generator. On-Off Keying (OOK) was used as a modulation scheme, which means that the lights were either at nominal intensity or off. OOK is easy to implement and decode at the receiver side. Other methods such as Orthogonal Frequency Division Multiplexing (OFDM) are less prone to inter-symbol-interference, at the cost of increased computational complexity [26]. Table I shows an overview of the LED parameters. Listed frequencies are obtained as the frequency supplied by the signal generator, transmitter positions were measured with a ruler.

A Kobuki robot [27] was selected as the mobile platform (see Fig. 2). The robot had a gyroscope and encoders built-in, which provided the odometry data. The OpenMV M7 camera was selected as the image sensor, due to the high degree of control it allows [28]. Finally, a 2D laser scanner was placed on the upper platform of the robot. More specifically, the RPLidar A1 by SLAMTEC was used. Data from this sensor can be used by Simultaneous Localization And Mapping (SLAM) algorithms to reconstruct a map of the environment, as well as the followed trajectory. A relatively large number of algorithms have been proposed to solve the SLAM problem, some of which are open source and freely available [29]. In this work, we used the Google Cartographer SLAM algorithm to reconstruct the ground truth trajectory of the robot [30]. The algorithm has been shown to provide centimeter accuracy for small environments, even with low-cost sensors [30], [31]. Other experimental positioning systems proposed in literature have also successfully used it as a benchmark [32], [33].

The robot was driven manually via a remote control, which relayed velocity commands through a laptop connected to the robot. The different sensors are also connected to this laptop that records the data. Position estimation was performed in post-processing. Therefore, the experimental results
TABLE I: LED Modulation Frequencies and Positions

\begin{tabular}{lll}
\hline Light source & Frequency $[\mathbf{k H z}]$ & Position $(\mathbf{x}, \mathbf{y}, \mathbf{z})[\mathbf{m}]$ \\
\hline LED 1 & 1.57 & $(0.0,0.0,1.50)$ \\
LED 2 & 2.03 & $(0.0,1.62,1.50)$ \\
LED 3 & 2.87 & $(1.78,1.63,1.50)$ \\
LED 4 & 4.92 & $(1.78,0.0,1.50)$ \\
\hline
\end{tabular}

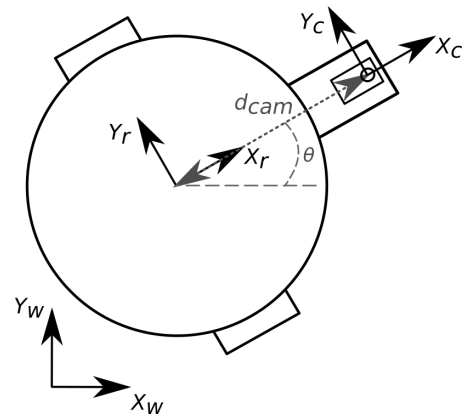

Fig. 3: Robot, camera and world coordinate frames.

are not real-time positioning results. However, the proposed algorithm is not very costly from a computational point of view (see Section III). The choice to perform estimation in post-processing was mostly motivated by the fact that this speeds up development time of the filter (but not necessarily its runtime) significantly, as data can be recorded once and then used repeatedly. Comparable research performed evaluations based on recorded data in a similar way [19]. We include all the pre-processing time when calculating the latency. In the real-time case, the latency could be limited by the rate at which the sensors return data. However, even in that case, a low processing latency is preferable, as it frees up resources for other activities (e.g., path planning and navigation). Another advantage to post-processing, is that it allows us to evaluate the different filters on the exact same inputs, thereby enabling a comparison between them that is less prone to outliers.

Most processing was performed on a Dell Latitude E5550 laptop. Additionally, to highlight the efficiency of our approach, we also performed position estimation on a Raspberry $\mathrm{Pi}(\mathrm{RPi})$, using the same algorithms and data. A Raspberry $\mathrm{Pi}$ is a low power single-board computer. The low cost and small form factor of the board make it well suited for a variety of applications. However, it has a relatively low amount of computational power. The main specifications of the hardware used in experiments and processing can be found in Table II.

\section{B. Extended Kalman Filter}

In this paper, we employed an EKF, which is an algorithm that can be used for state estimation of dynamic systems. The state in this case refers to the pose (position and orientation) of a mobile robot. The first step in the EKF (also known as the "prediction step"), is to predict the next state based on the previous state and a state-transition model (also known as the "motion model"). For our application, the motion model estimates the next pose of the robot based on odometry measurements [34]. Next, the Kalman filter takes a state dependent-measurement as an input. Based on a so called "measurement model", the observation at the predicted state is 
TABLE II: Overview of the Main Hardware Components and Parameters

\begin{tabular}{|c|c|c|}
\hline Description & Value & Unit \\
\hline \multicolumn{3}{|c|}{ Filter parameters } \\
\hline Initial uncertainty on $x$-coordinate & 0.5 & $\mathrm{~m}$ \\
\hline Initial uncertainty on $y$-coordinate & 0.5 & $\mathrm{~m}$ \\
\hline Initial uncertainty on heading angle & 30 & $\circ$ \\
\hline $\begin{array}{l}\text { Odometry distance } \\
\text { measurement uncertainty }\end{array}$ & $6 \times 10^{-5}$ & $\mathrm{~m}$ \\
\hline $\begin{array}{l}\text { Odometry angle } \\
\text { measurement uncertainty }\end{array}$ & $5 \times 10^{-4}$ & $\mathrm{rad}$ \\
\hline $\begin{array}{l}\text { Light source } \\
\text { measurement uncertainty }(x)\end{array}$ & 5 & pixels \\
\hline $\begin{array}{l}\text { Light source } \\
\text { measurement uncertainty }(y)\end{array}$ & 5 & pixels \\
\hline Number of particles & 250 & \\
\hline Particle filter convergence radius & 0.2 & $\mathrm{~m}$ \\
\hline \multicolumn{3}{|c|}{$\begin{array}{l}\text { Camera specifications } \\
\end{array}$} \\
\hline Resolution & $640 \times 480$ & pixels \\
\hline Focal length & 2.8 & $\mathrm{~mm}$ \\
\hline Frame rate & 30 & frames $/ s$ \\
\hline \multicolumn{3}{|c|}{ LIDAR specifications } \\
\hline Angular resolution & 1 & o \\
\hline Distance resolution & 0.2 & $\mathrm{~cm}$ \\
\hline \multicolumn{3}{|c|}{ Robot specifications } \\
\hline Gyroscope measurement range & 110 & $0 / s$ \\
\hline Odometry resolution & 2578.33 & tics/revolution \\
\hline \multicolumn{3}{|c|}{ Laptop specifications } \\
\hline CPU clock speed & 2.3 & $\mathrm{GHz}$ \\
\hline RAM & 8 & GB \\
\hline \multicolumn{3}{|c|}{ Embedded board specifications } \\
\hline CPU clock speed & 1.2 & $\mathrm{GHz}$ \\
\hline RAM & 1 & GB \\
\hline
\end{tabular}

calculated. The difference between the real and the predicted measurement, is new information (also called the "innovation"), which is weighed with the predicted state to obtain a new estimate. In this work, the (predicted) measurements consist of the pixel coordinates of a light source. Section II-E details the image processing pipeline, which produces the measurement vector. The observation model used to obtain the predicted measurements is described in Section II-F. The coordinates of the first detected light source were used as the initial position estimate. From this point onward, it was not necessary to continuously have a light source in view. A common issue with the EKF stems from data association problems. That is, the wrong (predicted) measurement is used in the calculation of the innovation. This may be caused by an error on the state estimate, or sensor failing. One can establish the correctness of the innovation by checking the uncertainty. This is also known as an innovation bounds test [35]. When the innovation is too large compared to the uncertainty, we reject the update and only use the prediction step of the EKF, thereby avoiding erroneous innovations

\section{Particle filter}

Like the EKF, the PF is an algorithm based on Bayes' theorem that is often used for state estimation and sensor fusion. Contrary to the EKF, the PF tracks the position of many possible estimates (called "particles") over time. Each particle is assigned a weight based on its likelihood. The PF effectively takes many samples of the measurement probability, rather than assuming a normal distribution. This enables it to model virtually any distribution (given enough particles).
In the first iteration, all particles of our PF were assigned identical weights. Their position is a Gaussian distribution, the center of which is the position of the first detected light source. Following initialization, the filter operates according to the following steps [36]:

1) Prediction: Similar to the EKF, odometry measurements are used to predict the next state of each particle. Particles are also slightly dispersed according to the odometry uncertainty (Table II) to avoid filter degeneracy.

2) Updating weights: Based on the predicted particle pose, pixel coordinates of the light source are obtained. Particles are then assigned a new weight based on how well this predicted measurement matches the observation. Measurements and predicted measurements are obtained in the same way as the EKF (see Sections II-E and II-F).

3) Resampling: Iteratively performing the prediction and update step may still lead to filter degeneracy. Therefore, most PF implementations perform resampling, whereby particles with lower probability are replaced by copies of particles with a high weight. In this work, we used the systematic resampling method [37], which ensures that samples are drawn from the entire particle space, and that larger weights are sampled more often. The method has been used extensively in literature, and works well across a variety of problems [36].

4) Estimating pose and covariance: The overall estimate is obtained as the weighted average of all the particles.

\section{Combined filter}

The EKF can provide relatively good results and is not computationally expensive. On the other hand, the filter does not perform well for highly non-linear problems. Compared to the EKF, the PF is generally more accurate and robust, at the cost of computational power [36]. We propose a hybrid particle/Kalman (PaKa) filter, which aims to combine the best of the two algorithms, similar to [21], [22]. Following the first light detection, estimation is initially performed with the PF. Every iteration, we verify whether the particles are all within a certain radius. When the particle cloud converges, estimation continues with an EKF, which is initialized with the current estimate of the PF. If, during EKF estimation, the innovation bounds test fails, estimation continues with a PF. The initial particle cloud is in this case a Gaussian distribution defined by the current estimate.

\section{E. Image processing pipeline}

Most camera-based VLP systems rely on the rolling shutter principle. CMOS cameras expose and read out the lines of the sensor sequentially, which can lead to unwanted effects such as skewed objects when the camera is moving. However, in VLP systems one can exploit this principle to sample optical signals at much higher rates than the camera frame rate. Each horizontal line in the image effectively becomes a sample (see Fig. 4), the sampling rate is therefore given by:

$$
f_{s}=n_{h} F P S
$$




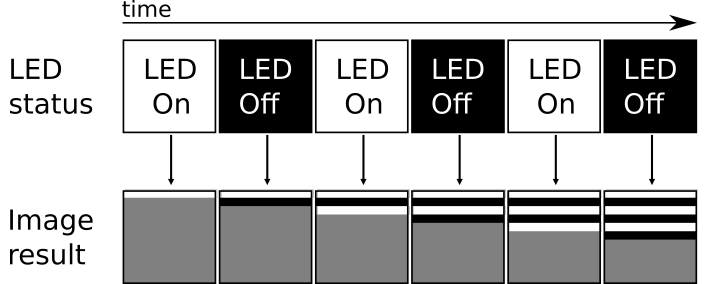

Fig. 4: Rolling shutter principle [38].

where $f_{s}$ is the sampling rate, $F P S$ is the number of frames per second returned by the camera, and $n_{h}$ is the number of horizontal lines in an image.

After an image is taken, lens distortion is first removed. To this end, we performed a one time calibration procedure with the method published by Zhang [39]. Some residual distortion does remain at the edges of the image, therefore we discard detected light sources that are too far from the center. Following distortion removal, the light source location and frequency are calculated via the image processing pipeline detailed in our previous work [38]. The process is illustrated in Fig. 5. First, a Gaussian blur is applied to the image to remove the stripe pattern. Then, a binary threshold is used to single out the pixels belonging to the light source. Reflections have a lower intensity in the image, so they are generally removed in this step. Next, the contour of the light source is detected and its center coordinates are calculated. These center coordinates are later used as the coordinates of the light source. The centroid coordinates are also used to crop the original image. A Gaussian blur is again applied to this cropped image, this time with a kernel that is 1 pixel high to smear out the stripe pattern and make it easier to detect. Canny edge detection [40] is used to detect the transitions between white and dark stripes. Finally, with the number of pixels between each transition and the line readout time, the modulation frequency of the source is calculated.

\section{F. Measurement model}

The measurement model predicts perfect sensor measurements based on the predicted state. For our work, this means that the model predicts pixel coordinates of an LED based on the predicted robot pose. The real positions and modulation frequencies of the light sources were measured beforehand, and saved in a lookup table. The first step in the measurement model matches the modulation frequency calculated by the image processing pipeline (Section II-E) to the real frequency of one of the light sources. The frequency that provides the best match is used. However, the difference between the real and the detected frequency should be below a certain threshold.

Knowing the world coordinates of the light source, the pixel coordinates can be calculated by applying the pinhole camera model [41]. First, the coordinates of the light source are expressed in homogeneous coordinates:

$$
\boldsymbol{X}_{l, \boldsymbol{w}}=\left[\begin{array}{llll}
x_{l, w} & y_{l, w} & z_{l, w} & 1
\end{array}\right]^{T}
$$

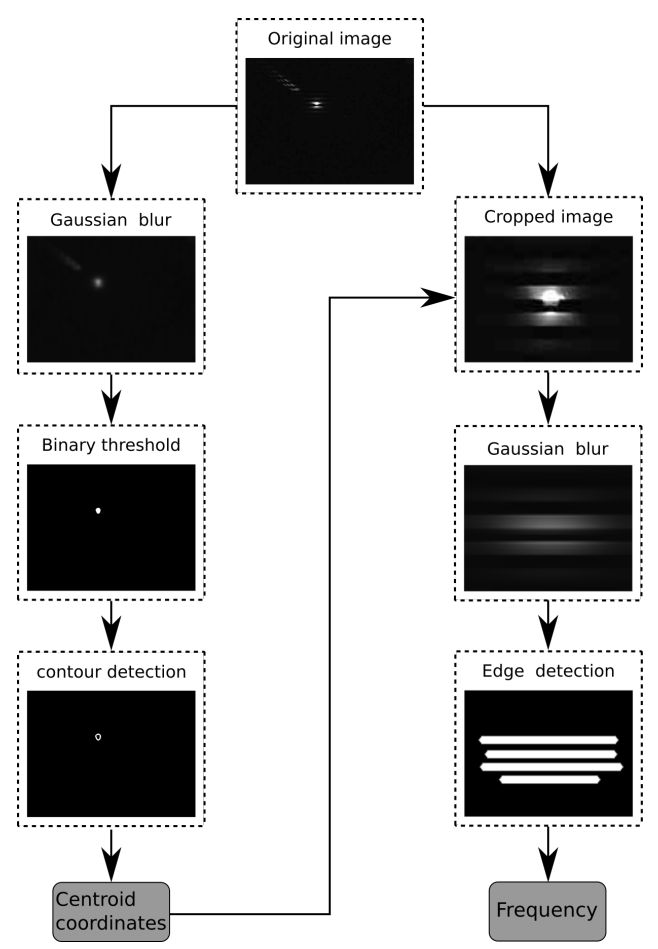

Fig. 5: Image processing pipeline [38]

where $\boldsymbol{X}_{l, \boldsymbol{w}}$ is the position vector of the light fixture in the world frame and $x_{l, w}, y_{l, w}$ and $z_{l, w}$ are the $x$ - $y$ - and $z$ coordinates of the light source in the world frame, respectively. The transformation between the world frame and the camera frame is described by the following matrix:

$$
\boldsymbol{T}_{\boldsymbol{C}, \boldsymbol{w}}=\left[\begin{array}{cccc}
\cos \left(\theta_{r, w}\right) & -\sin \left(\theta_{r, w}\right) & 0 & x_{c a m, w} \\
\sin \left(\theta_{r, w}\right) & \cos \left(\theta_{r, w}\right) & 0 & y_{c a m, w} \\
0 & 0 & 1 & z_{c a m, w} \\
0 & 0 & 0 & 1
\end{array}\right]
$$

where $\boldsymbol{T}_{\boldsymbol{C}, \boldsymbol{w}}$ is the transformation matrix from the camera to the world frame, $x_{c a m, w}, y_{c a m, w}$ and $z_{c a m, w}$ are the $x$ - $y$ - and $z$-coordinates of the camera in the world frame, respectively. The camera coordinates can be recovered from the robot pose and the distance between the camera and the robot center:

$$
\begin{aligned}
& x_{c a m, w}=x_{r, w}+d_{c a m} \cos \left(\theta_{r, w}\right) \\
& y_{c a m, w}=y_{r, w}+d_{c a m} \sin \left(\theta_{r, w}\right)
\end{aligned}
$$

where $d_{\text {cam }}$ is the distance between robot center and the camera sensor (see Fig. 3). By applying the inverse transformation, we transform the light source from the world coordinate frame to the camera coordinate frame:

$$
X_{l, c}=T_{C}^{-1} X_{l, w}
$$

where $\boldsymbol{X}_{l, c}$ is the position vector of the light coordinates in the camera frame. These coordinates are currently expressed in physical units (e.g., meters), and can be converted to pixel units by projecting them with the camera matrix [41]:

$$
\boldsymbol{X}_{l, i}=\left[\begin{array}{llll}
x_{l, i} & y_{l, i} & z_{l, i} & 1
\end{array}\right]^{T}=\boldsymbol{C}^{-1}\left(\boldsymbol{T}_{\boldsymbol{C}}^{-1} \boldsymbol{X}_{\boldsymbol{w}}\right)
$$

where $\boldsymbol{X}_{l, i}$ is the position vector of the light source in the image frame, $x_{l, i}, y_{l, i}$ and $z_{l, i}$ are the $x$ - $y$ - and $z$ coordinates 
of the light in the image frame, respectively and $C$ is the camera matrix:

$$
\boldsymbol{C}=\left[\begin{array}{cccc}
f & 0 & 0 & x_{\text {center }} \\
0 & f & 0 & y_{\text {center }} \\
0 & 0 & 1 & 0 \\
0 & 0 & 0 & 1
\end{array}\right]
$$

where $f$ is the focal length of the camera and $x_{c e n t e r}$ and $y_{\text {center }}$ are the $x$ and $y$-coordinates of the optical center.

\section{G. Data processing and evaluation}

Most evaluations of indoor positioning systems report the average positioning error [1]. In this section, we will explain how we obtained the positioning error of the experiments. Besides accuracy, precision is an important evaluation metric for IPS. Ideally, a system has a low average error, and a low spread on the error values. To measure precision, the 95th percentile of the cumulative error distribution is often used [1][42], this is also referred to as the P95 value. Finally, latency is another important evaluation metric. Similar to [19], we obtain the latency by dividing the total processing time for an experiment by the number of estimates.

Following experiments, the recorded data were loaded into memory and processed sequentially. An overview of the processing steps is shown in Fig. 6. Once a light source was identified, the position of this transmitter in the world frame was used as the initial estimate of the robot position. Next, the estimation algorithms looped over all measurements and estimated the robot pose. However, the odometry sensors provided data at higher rates than the camera. Therefore, both steps of the filters were only performed when the timestamps of all data were within $0.1 \mathrm{~s}$ of each other (interpolation was not used). Otherwise, only the prediction step was used.

The positioning algorithms and Google Cartographer also produced position estimates at different rates. To calculate the positioning error, these trajectories were first time synchronized. Again, only data points that had timestamps within 0.1 seconds of each other were considered. Additionally, the Cartographer trajectory was defined relative to the robot starting position, whereas the trajectory returned by our algorithm was defined relative to the light sources. It is challenging to obtain an accurate transformation between these two frames. Therefore, we used the Iterative Closest Point (ICP) algorithm to find the optimal transformation between these two frames.

When the ground truth and the estimated positions have been synchronized in time and space, it is possible to calculate the average positioning error. The positioning error is defined as the Euclidean distance between the true position and the estimated position:

$$
\varepsilon_{R}=\sqrt{\left(x_{\text {est }}-x_{\text {real }}\right)^{2}+\left(y_{\text {est }}-y_{\text {real }}\right)^{2}}
$$

where $\varepsilon_{R}$ is the positioning error and subscripts est and real are used to denote the estimated and the real coordinates, respectively.

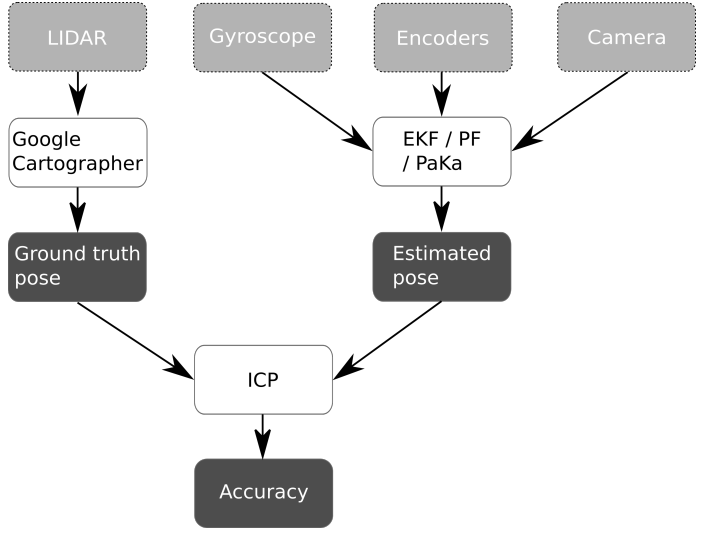

Fig. 6: Processing steps to obtain positioning accuracy.

TABLE III: Baseline Experimental Results

\begin{tabular}{lllll}
\hline Filter & $\begin{array}{l}\text { Mean } \\
\text { accuracy }[\mathbf{m}]\end{array}$ & $\begin{array}{l}\text { P95 } \\
{[\mathbf{m}]}\end{array}$ & $\begin{array}{l}\text { Average latency } \\
\text { (laptop) }[\mathbf{m s}]\end{array}$ & $\begin{array}{l}\text { Average latency } \\
(\mathbf{R P i})[\mathbf{m s}]\end{array}$ \\
\hline $\mathrm{EKF}$ & 0.040 & 0.091 & 5.6 & 71.7 \\
$\mathrm{PF}$ & 0.025 & 0.046 & 7.9 & 82.3 \\
$\mathrm{PaKa}$ & 0.023 & 0.035 & 5.9 & 74.4 \\
\hline
\end{tabular}

\section{RESULTS}

\section{A. Baseline results}

During experiments, the robot always starts in the top left of the setup (see Fig. 1). Initially, it drives forward in an approximately straight line. Upon reaching the edge of the setup, it turns 180 degrees and continues in the other direction. The rest of the space is covered in a zigzag pattern (see Fig. 7). This trajectory ensures that some light observations will be made, yet also leads to a period where no light sources are visible (e.g., in the middle of the test space). Three experiments were performed under the same conditions, and the accuracy with respect to the ground truth was calculated by the procedure outlined in Section II. The results for each experiment are averaged to obtain the overall accuracy. For these baseline results, the blinds of the windows were closed to limit interference from ambient lighting. Table III provides an overview of the main results for each filter.

Fig. 7a shows the results of the EKF algorithm, alongside the ground truth. It is clear that estimation only starts when the robot approaches the first light source. As the trajectory continues, the position initially remains very close to the ground truth. Around the center of the space, the orientation (and consequently the position) starts to drift. This drift is corrected as a new observation of a light source is made. Towards the end of the experiment, a smaller correction is also visible. Fig. 7d shows the error with respect to the ground truth as a cumulative distribution. This distribution contains the errors from all experiments. As listed in Table III, the mean accuracy is around $4 \mathrm{~cm}$, with $95 \%$ of errors being below approximately $9 \mathrm{~cm}$. Additionally, latency is relatively low at $5.6 \mathrm{~ms}$. Even when using the RPi, the algorithm is able to estimate the robot position approximately 14 times per second.

Fig. $7 \mathrm{~b}$ shows the pose estimate of the PF. Contrary to the EKF, there is an observable transition period, during which the filter estimate needs to converge to the true position. During 


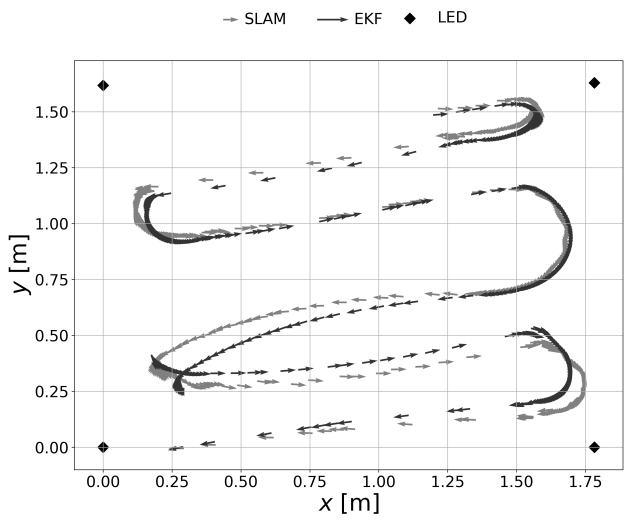

(a) EKF estimate.

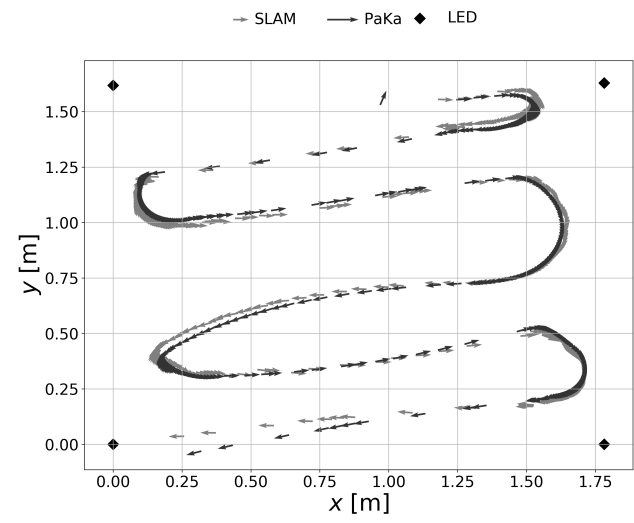

(c) Combined filter estimate.

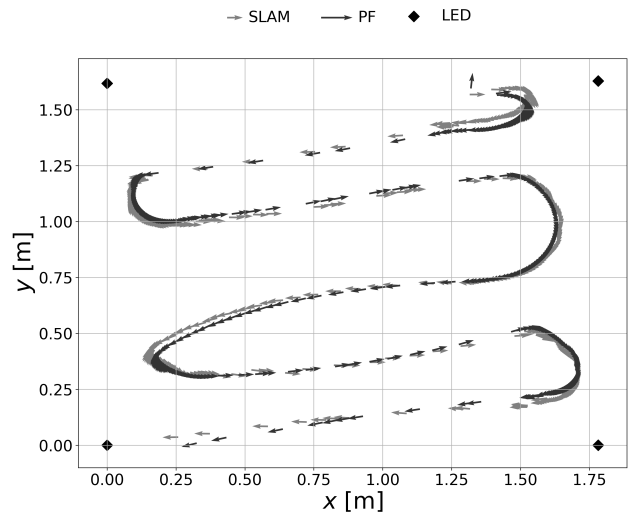

(b) PF estimate.

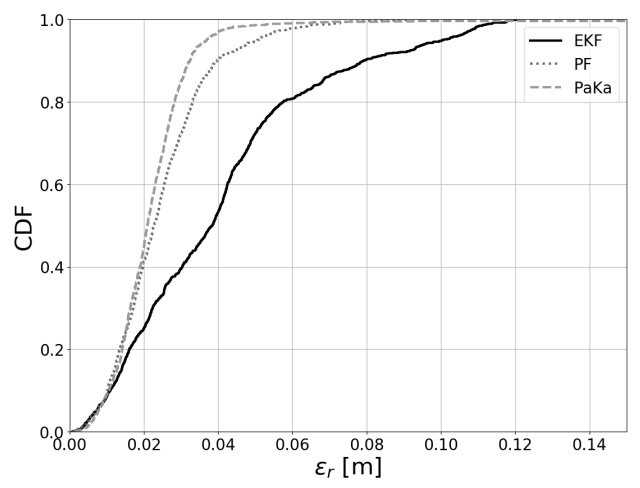

(d) Cumulative error distribution.

Fig. 7: Filter estimates for a zigzag experiment.

the first two evaluation points of the PF, the particle cloud is relatively spread out according to the initial uncertainty, therefore the estimate is initially not very accurate. From the $3^{\text {rd }}$ iteration onward, accuracy significantly improves. Similar to Fig. $7 \mathrm{a}$, the angle estimate also starts to drift around the center of the space, yet to a lesser extent. Following the observation of a light source, drift is again corrected. Because a smaller correction was necessary compared to the EKF, the overall accuracy improves. This is also visible in the cumulative distribution (Fig. 7d). On average, the accuracy of the PF across all experiments is $2.5 \mathrm{~cm}$, with a $\mathrm{P} 95$ value of 4.6 $\mathrm{cm}$, which is significantly better than the EKF. Interestingly, the effect of the initial iterations on the overall accuracy is relatively low. When the first 3 estimates are removed from the accuracy calculations, the result changes only one or two millimeters. Due to the large number of estimates during the trajectory, the effect is averaged out. The main drawback of the PF, is that the latency is increased compared to the EKF. For this trajectory, however, the increase is relatively limited (approximately 2 and $10 \mathrm{~ms}$ for the laptop and the $\mathrm{RPi}$, respectively). Using the code profilers available in the Python programming language, it is possible to determine which parts of the program take the longest time to execute. Timing results for all filters are shown in Fig. 8. In total, it took approximately 3 minutes to process all data from both trajectories (see Section III-C) with the PF. Approximately two-thirds of that time was spent on estimating the robot pose. The vast majority of estimation time was spent on updating the weights of the particles. In contrast, the EKF was able to process the data much faster (see Fig. 8), as the costly weight updates are not performed in the algorithm. Consequently, most of the processing time is spent on processing the image and odometry data.

Estimates of the combined filter are shown in Fig. 7c. Because the filter is initialized as a PF, the first few iterations are again not very accurate. Following initialization, the estimate is very similar to the PF, yet accuracy is slightly lower for this specific example. However, if we consider all experiments (Fig. 7d and Table III), the PaKa filter is actually more accurate than both the EKF and the PF. This implies that the filters complement each other well. When profiling the code of the PaKa filter (Fig. 8), we find that, like the EKF, the majority of the time is spent on processing the measurement data, rather than estimating the position. Compared to the PF results in Fig. 8, the costly operations of weight updating are performed more sparingly. Most of the iterations are actually performed with the EKF, only at initialization (and occasionally when drift occurs) Monte-Carlo methods are used. This provides better initialization for the EKF, and therefore higher accuracy. Yet due to the limited number of times that particles are 


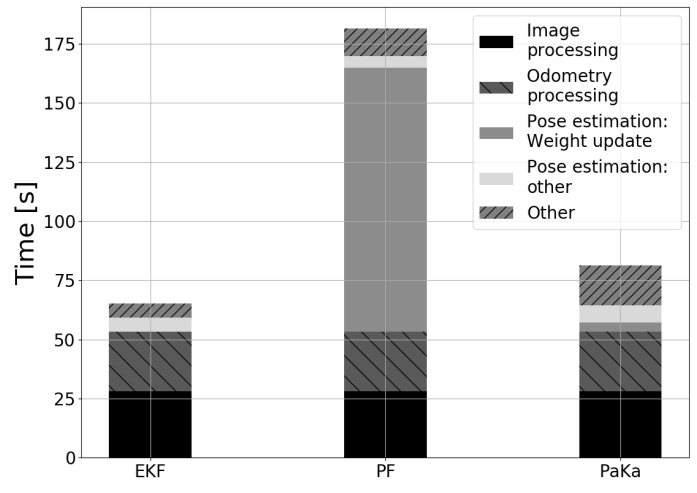

Fig. 8: Time spent in different portions of the algorithms.

propagated, the update rate can be almost as high as the EKF. Therefore, we obtain a hybrid filter that is more accurate and robust by making a small trade-off on the update rate.

\section{B. Variability of results}

Due to varying background processes on the computing platforms, the update rates of the filters can vary. Additionally, the prediction step of the PF slightly disperses the particles to avoid filter degeneracy. As a result, there is a random component in estimations performed by the particle and hybrid filter. Accuracy can therefore vary, as certain predictions may disperse the particles closer or further from the true position. This effect is reduced by averaging over the data from multiple experiments. Accuracy of the EKF is unaffected as no probability distributions are sampled.

To determine the variability on the results obtained in the previous section, we process data from the same experiment 100 times sequentially with each filter. Accuracy and timing results are saved for each iteration. Fig. 9 visualizes the range of obtained latencies as a cumulative distribution. It is clear that the processing times of the laptop are relatively stable. For the EKF, $99 \%$ of results are within a $0.2 \mathrm{~ms}$ range. However, the first iteration could be processed significantly faster $(5 \mathrm{~ms}$ compared to $5.35 \mathrm{~ms}$ on average). For the PF, virtually all iterations are within a $0.25 \mathrm{~ms}$ range. Again, only the first result deviates significantly. On the other hand, processing times for the combined filter have a larger spread. In order to contain $97 \%$ of results, a $0.4 \mathrm{~ms}$ range is needed. The random nature of particle dispersion leads to convergence of the cloud, and therefore a switch to the EKF, at different times, leading to a higher variability in processing times. Similar observations can be made when performing the computations on the Raspberry Pi. However, results are more spread out in this case, which can likely be attributed to thermal throttling. Contrary to the laptop, the RPi has no active cooling. When the temperature rises due to increased CPU activity, the chip limits clock rates in order to avoid overheating [43]. Therefore, clock speed of the RPi is inherently less stable. Nevertheless, our overall observations from before still apply.

For the PF, 97\% of mean accuracy results are within $1 \mathrm{~cm}$. The P95 values are more spread out, and 97\% of observations are within $5 \mathrm{~cm}$. In a few cases, significantly higher errors

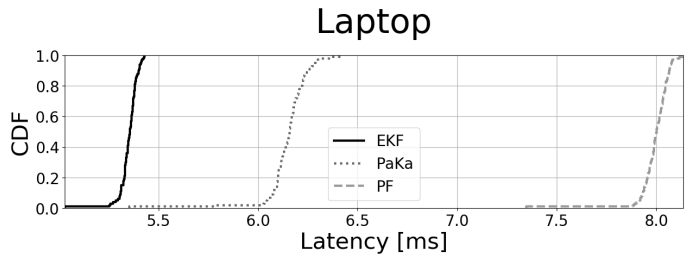

$\mathrm{RPi}$

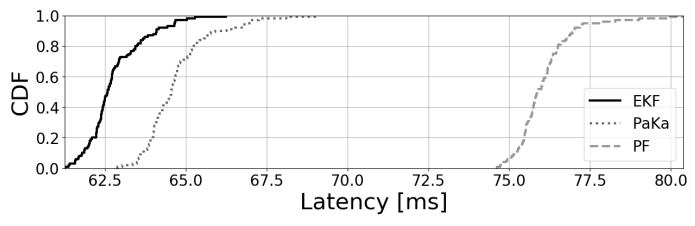

Fig. 9: Variability of positioning latency.
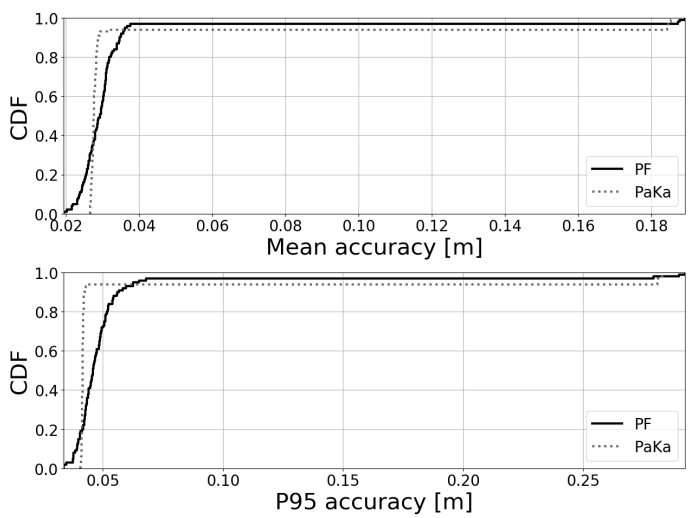

Fig. 10: Variability of accuracies.

TABLE IV: Experimental Results for Stop-Go Trajectory

\begin{tabular}{lllll}
\hline Filter & $\begin{array}{l}\text { Mean } \\
\text { accuracy }[\mathrm{m}]\end{array}$ & $\begin{array}{l}\text { P95 } \\
{[\mathbf{m}]}\end{array}$ & $\begin{array}{l}\text { Average latency } \\
\text { (laptop) }[\mathrm{ms}]\end{array}$ & $\begin{array}{l}\text { Average latency } \\
\text { (RPi) }[\mathrm{ms}]\end{array}$ \\
\hline $\mathrm{EKF}$ & 0.030 & 0.060 & 6.6 & 72.4 \\
$\mathrm{PF}$ & 0.030 & 0.057 & 21.2 & 152.1 \\
$\mathrm{PaKa}$ & 0.022 & 0.040 & 7.2 & 75.4 \\
\hline
\end{tabular}

can be observed, which can be attributed to inaccuracies in ICP matching. The trajectory estimated by the filter was not significantly different in this case, yet a poor match was calculated, resulting in large positioning errors. When this occurs in subsequent sections, we simply process the data again until a match is found. Because the majority of estimations are performed by the EKF, results of the PaKa filter are clustered more closely together. Less than half a centimeter is needed to contain the vast majority of mean and P95 accuracy results. Occasionally, the ICP algorithm again encounters issues when matching the trajectories.

\section{Additional measurements}

The results of the previous sections were obtained with only a few discrete corrections to the trajectory, which are especially noticeable for the EKF. We now hypothesize that additional measurements would improve this accuracy further. The most straightforward way to increase the number of measurements, would be to increase the number of light sources. However, in our setup it is quite challenging to increase the number of transmitters. A signal generator is 


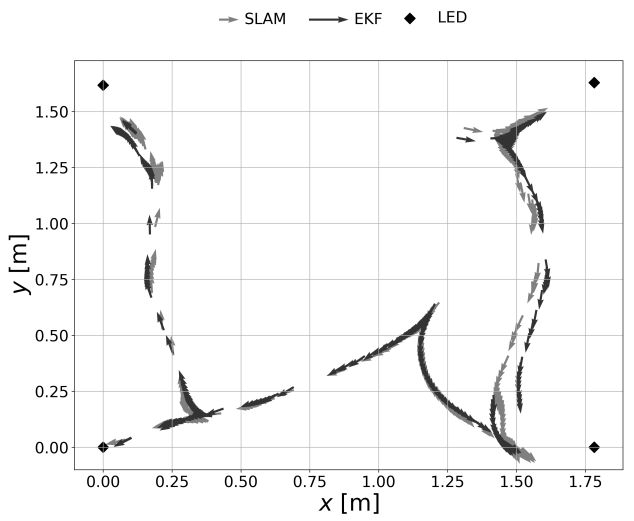

(a) EKF estimate.

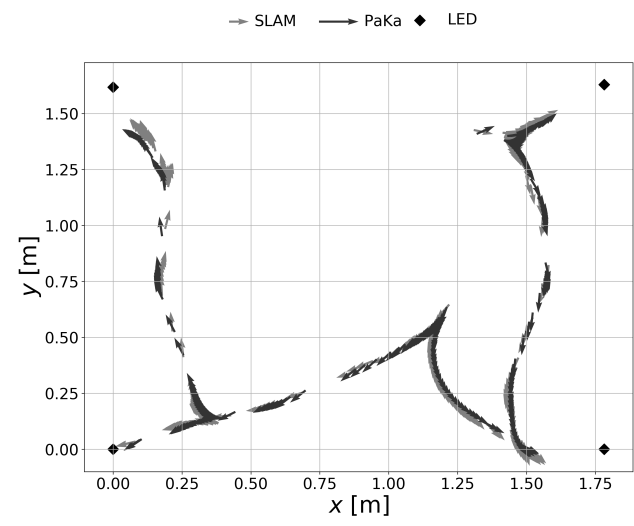

(c) Combined filter estimate.

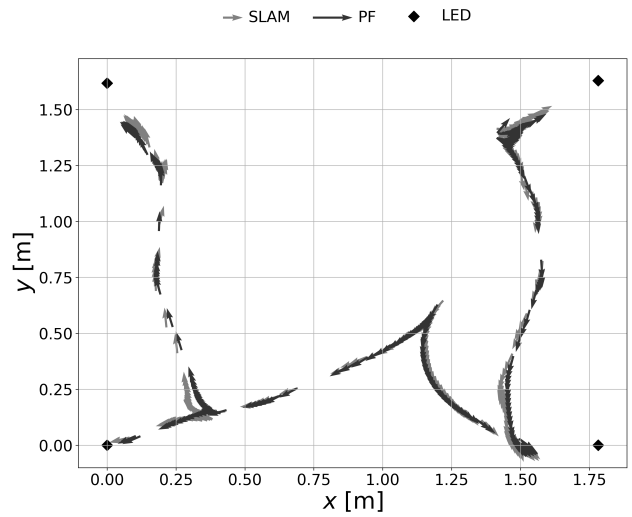

(b) PF estimate.

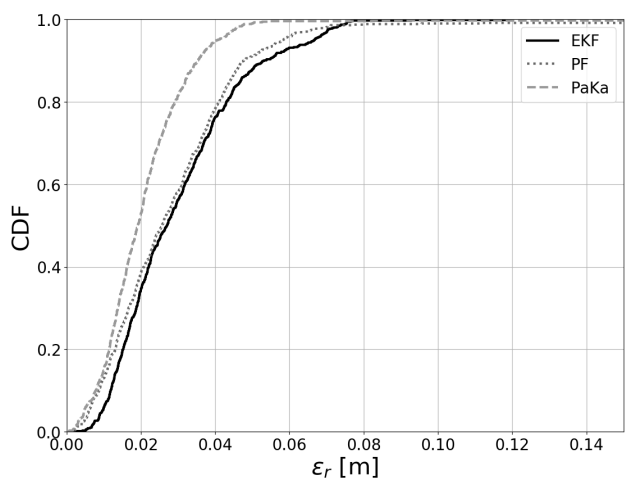

(d) Cumulative error distribution.

Fig. 11: Filter estimates for a stop-go experiment.

required for each individual source, in order to modulate its intensity. Unfortunately, such devices are rather expensive. An alternative way to artificially increase the number of measurements, is to drive the robot to each individual LED, and record data for a few seconds before continuing. We call this a "stop-go" experiment. An example (for the EKF) is shown in Fig. 11a. Similar to Fig. 7a, estimation begins close to the first observed light source. Additionally, orientation drift is corrected as a new source is observed. The accuracy is again shown as a cumulative distribution in Fig. 11d. It is clear that, for the EKF, the overall accuracy is higher with these additional measurements. The mean error is slightly lower than the zigzag trajectory at $3 \mathrm{~cm}$. Larger improvements are mostly visible in the higher percentiles of the distribution, as is reflected in the P95 value of $6 \mathrm{~cm}$. However, introducing additional measurements does result in an additional delay, which is much more pronounced for the laptop than the RPi. A larger number of images need to be processed, therefore the update rate is also lower compared to the zigzag trajectory (see Table IV). Nevertheless, the delay remains quite low.

Interestingly, the PF (Fig. 11b) shows a small decrease in accuracy with these extra measurements, but the change is within the margin of error defined in the previous section. As can be seen in Fig. 7d, the PF is still more accurate than the EKF, yet only slightly, indicating that PF accuracy is more robust to a changing number of measurements. However, the influence on the update rate is much larger for the PF. Where EKF processing times increased slightly, the PF is now more than 2.5 times slower on the laptop. The RPi is only able to make roughly 6 estimates per second with the PF. With the increased number of observations, the computationally intensive tasks of weight updating and resampling need to be performed more often, thereby increasing the processing time.

Similar to section III-A, the combined filter (Fig. 11c) has better accuracy than the EKF and the PF. Like the PF, positioning results remained relatively stable even with an increased number of observations. On the other hand, the update rate changed similar to the EKF. Indeed, the laptop processing times were increased by approximately $23 \%$. Processing times on the RPi were increased only slightly. The combined filter was thus impacted slightly more than the standard EKF, yet much less than the PF.

\section{Robustness to Changing Environmental Conditions}

Previous experiments were all performed in the same environment, under the same conditions. However, it is important that the system can also operate under different circumstances. Most notably, changing lighting conditions tend to have an adverse effect on computer vision. In this section, we change a number of environmental parameters, and compare the results 
TABLE V: Accuracy Results for Different Environmental Conditions (all values are expressed in meters)

\begin{tabular}{lllll}
\hline Dataset & Baseline & $\begin{array}{l}\text { Other } \\
\text { day }\end{array}$ & $\begin{array}{l}\text { Shutters } \\
\text { open }\end{array}$ & $\begin{array}{l}\text { Fluorescent } \\
\text { lights }\end{array}$ \\
\hline Zigzag (avg, EKF) & 0.040 & 0.030 & 0.032 & 0.021 \\
Zigzag (avg, PF) & 0.025 & 0.176 & 0.036 & 0.021 \\
Zigzag (avg, PaKa) & 0.023 & 0.023 & 0.020 & 0.021 \\
\hline Zigzag (P95, EKF) & 0.091 & 0.061 & 0.062 & 0.036 \\
Zigzag (P95, PF) & 0.046 & 0.551 & 0.070 & 0.042 \\
Zigzag (P95, PaKa) & 0.035 & 0.043 & 0.036 & 0.045 \\
\hline Stop-go (avg, EKF) & 0.030 & 0.034 & 0.035 & 0.026 \\
Stop-go (avg, PF) & 0.030 & 0.035 & 0.030 & 0.032 \\
Stop-go (avg, PaKa) & 0.022 & 0.032 & 0.030 & 0.028 \\
\hline Stop-go (P95, EKF) & 0.060 & 0.073 & 0.082 & 0.049 \\
Stop-go (P95, PF) & 0.057 & 0.071 & 0.053 & 0.059 \\
Stop-go (P95, PaKa) & 0.040 & 0.050 & 0.053 & 0.046
\end{tabular}

to the baseline obtained in previous sections. The different test cases (shown in Fig. 12) are:

- Other day: in order to verify that filter parameters are not overfit to a specific moment in time, additional experiments were performed on a different date. These measurements took place approximately three months after those discussed previously. The setup on this date is shown in the second row of Fig. 12. Compared to the baseline, it was still light outside, as this date was in the summer. Previous evaluations took place in early spring when the sun sets earlier.

- Shutters open: in order to simulate sunlight interference. This is an especially challenging use case in the summer, when the sky is less cloudy. These measurements took place on the later date in the summer as well. The setup is shown on the third row of Fig. 12.

- Fluorescent lights on: in order to verify that the system can cooperate with existing lighting infrastructure if needed. Often this is still fluorescent lighting, especially in older buildings. For these experiments, the blinds were also left open. Again, these measurements took place 3 months after the baseline experiments. The setup in this test scenario is shown on the final row of Fig. 12.

The main results are collected in Table V. For the sake of brevity, only the mean and P95 accuracy values are listed, rather then the complete distribution. Similar to previous sections, these values were obtained by performing each experiment three times, and averaging the resulting accuracies.

Both the EKF and the PaKa filter performed very similar on the later date compared to the baseline results. Only the P95 accuracy for the EKF on the zigzag trajectory is significantly different ( $6.1 \mathrm{~cm}$ compared to $9.1 \mathrm{~cm}$ for the baseline). Such an improvement is not present for the stop-go trajectory, in fact a slight decrease can be observed. Overall, performance for the Kalman and combined filter can be considered relatively stable. The PF on the other hand, performs significantly worse on the other day. This can likely be attributed to the shutters (see Fig. 12), which were closed on both days. However, the sunlight was much brighter on the later date which leads the shutters to now also produce a stripe pattern in the image (right picture on the second row of Fig. 12). Contrary to the reflections on the metal poles, these patterns are not removed by the threshold step in the image processing pipeline. There-

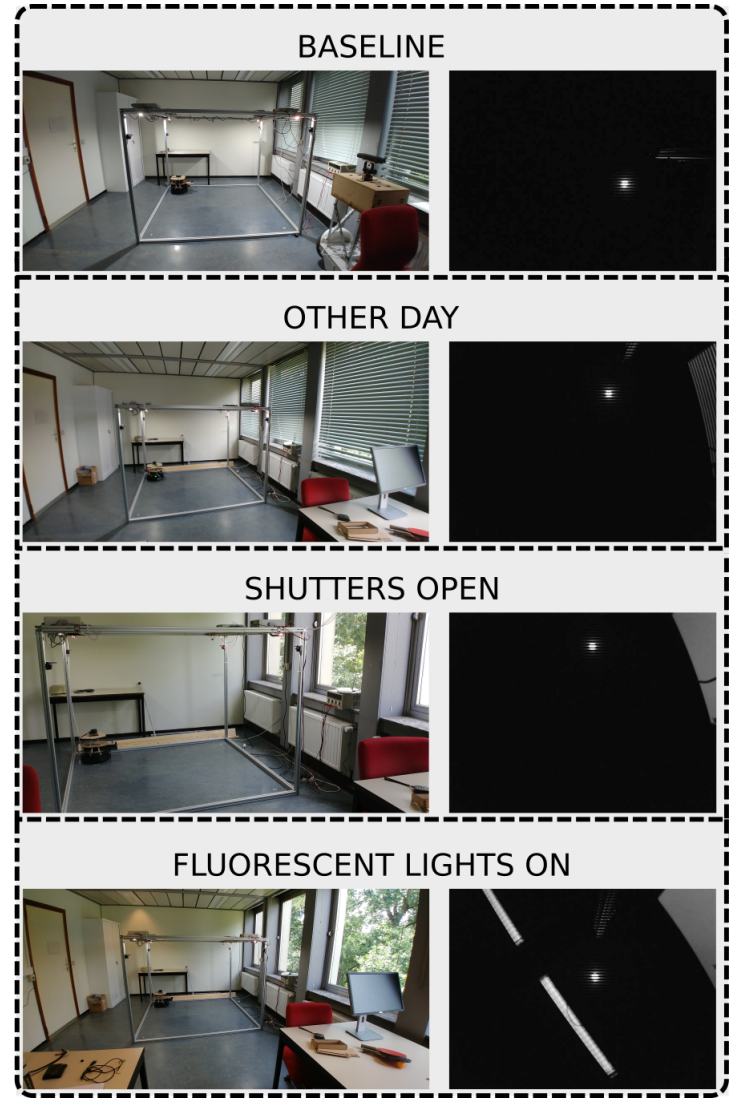

Fig. 12: Left column: Experimental setup under different conditions. Right column: Example picture taken by the camera.

fore, they are also detected as a light source. The EKF rejects this light source through the innovation bounds test, but our $\mathrm{PF}$ has no such compensation mechanism. This is especially problematic in the zigzag trajectory, where few light sources are observed. When more observations are made (e.g., in the stop-go trajectory), the estimate does not deteriorate.

From Table V, it is clear that opening or closing the blinds has almost no effect. Sunlight falling in through the window is not recognized as a light source, as it is not modulated. Consequently, it is simply ignored and there is little difference between the 'other day' and the 'blinds open' data. Only the PF benefits from opening the blinds, as now there are only stripe patterns originating from the LEDs.

Fluorescent light is modulated, yet at a much lower rate than our sources. Therefore, the system has no trouble filtering fluorescent lights as they have no stripe pattern in the image (lower right in Fig. 12). Interestingly, background lighting appears to even slightly improve the overall accuracy. Both the average and the P95 accuracies are lower compared to the other datasets. This may be explained by the influence of the ambient lighting on the stripe pattern. By adding a source of low-intensity light, the LEDs are still the primary light source. However, the white stripes have a larger contrast, as the ceiling now appears brighter due to the indirect light on it. In the current experimental construction, the area around the LEDs prevents light from falling on the same location. Therefore, the brighter stripes are brightened up, yet the dark stripes 
TABLE VI: Comparison of This Paper with Related Work

\begin{tabular}{|c|c|c|c|}
\hline Ref & Algorithm & $\begin{array}{l}\text { Accuracy } \\
{[\mathrm{cm}]}\end{array}$ & Latency [ms] \\
\hline [10] & Projection & $13.0-17.5$ & Not specified \\
\hline [11] & $\begin{array}{l}\text { Projection } \\
+\mathrm{IMU}\end{array}$ & 11.2 & Not specified \\
\hline [15] & Rolling shutter & $17.0-236.0$ & $1700-4000$ \\
\hline [16] & EKF & 14.5 & Not specified \\
\hline [17] & $\mathrm{PF}$ & 14.0 & Not specified \\
\hline [19] & PF & 2.98 & 20 \\
\hline This paper & EKF & $2.1-4$ & $6.1(\mathrm{PC})-72.1(\mathrm{RPi})$ \\
\hline This paper & $\mathrm{PF}$ & $2.1-17.6$ & $14.6(\mathrm{PC})-117.2(\mathrm{RPi})$ \\
\hline This paper & $\mathrm{PaKa}$ & $2.0-3.2$ & $6.6(\mathrm{PC})-74.9(\mathrm{RPi})$ \\
\hline
\end{tabular}

still receive little direct light (due to the short exposure time, indirect light does not register in the image sensor) leaving a better contrast. Overall, the effect is similar to a lampshade: the light emitted by the signal LED is reflected off a larger surface (the indirectly illuminated ceiling), which increases the area of the stripe pattern (more and longer stripes) and improves contrast. This effect is specific to our experimental setup, and may not necessarily appear elsewhere. Only the ambient fluorescent lights have this effect. As mentioned before, opening or closing the blinds has limited impact. Due to the angle of the sunlight, the ceiling is not illuminated. Consequently, sunlight is simply ignored.

Compared to the first round of experiments, the difference between the two trajectories is less pronounced. Often, the zigzag pattern even outperforms the stop-go trajectory, albeit by a very small margin. This may indicate that the zigzag already gathered sufficient data for accurate estimation. Overall, we can conclude that the system is robust under a range of lighting conditions. Additionally, the combined filter outperforms its components under nearly all conditions, whilst only introducing a small delay compared to the EKF.

\section{DISCUSSION}

The accuracy of our approach is comparable to some PDbased VLP systems, which can be in the range of centimeters [8]. Processing an image can take longer than a measuring and interpreting a voltage. However, our results show that the update rate can still be relatively high (up to $180 \mathrm{~Hz}$ ). Even on low-cost processing hardware, reasonable update rates can be achieved (up to $14 \mathrm{~Hz}$ ). Our approach may, therefore, be more interesting for devices that already have built-in cameras, as there is little trade-off to be made in terms of accuracy or delay. A comparison between this work and other VLP systems can be found in Table VI. By using the circular projection of LEDs, a relatively accurate location can be calculated with a single light in view [10], [11]. However, these works require an image resolution that is several orders of magnitude higher, and performed evaluations in a smaller environment. Moreover, their accuracy is substantially lower.

Compared to other sensor fusion approaches, our filters perform favorably. Accuracy is significantly higher than the lookup system [15], though our evaluation was performed in a much smaller environment. Similar to the VLIP system [18], our work can cope with intermittent outages. Additionally, it can operate under sparser lighting infrastructures and is evaluated with LED beacons (compared to AR tags used in [18]). In contrast, the system proposed in this paper cannot estimate a 3D position. Our EKF and PF compare positively to those proposed in [16] and [17], respectively. Our accuracy is higher overall, particularly in the higher percentiles of the cumulative distribution. The use of a camera over a photodiode provides more spatial information, and leads to higher accuracy. One would expect our processing times to be longer, yet we cannot make a direct comparison as the authors do not specify it. Additionally, we do not require multiple LEDs to be in view in order to update the position estimate. Therefore, the distribution of LEDs can be sparser. Note that we did test our system at a lower ceiling height, leading to a better signal to noise ratio. Of the previously discussed systems, our work is most similar to [19]. The authors also achieved an average accuracy of approximately $3 \mathrm{~cm}$, have a high update rate $( \pm$ $50 \mathrm{~Hz}$ ) and evaluated the robustness to partial shadowing and fluorescent light. The experimental setups used in our work and in [19] are similar in size. However, both are relatively small compared to other evaluations such as [15]. Contrary to our work, [19] did not investigate the influence of sunlight interference, which as we showed in section III, can cause problems for particle filters. Additionally, the shape and size of all LEDs needs to be identical and known in order to perform template matching Our approach only requires a stripe pattern be present in the image, which can be emitted by an LED of any shape. Finally, because of the lower resolution image sensor and the simpler fusion algorithm, the update rate of our approach is much higher with comparable processing hardware. It is even possible to perform position estimation with a low-cost embedded board.

The main drawback of our evaluation is the relatively small experimental setup. As the ceiling height increases, the stripe patterns in the images may be harder to detect, which in turn may force us to use a higher resolution camera after all. Alternatively, a lens with a smaller field of view (FOV) could be used, which would result in transmitters taking up a larger portion of the image. Consequently, stripes could be detected at larger distances. The approach outlined in this paper does not require multiple LEDs to be in view at all times, and would therefore likely perform well even with a smaller FOV. In future work, an evaluation in a larger indoor space is therefore needed to verify these hypotheses.

\section{CONClusion}

In this work, we described several ways to combine VLP data with odometry from a mobile robot. An EKF and PF were tested. Additionally, we proposed a hybrid particle/Kalman filter that combines the efficiency of the EKF with the accuracy of the PF. On average, accuracy of the tested filters is a few centimeters, and rarely exceeds $10 \mathrm{~cm}$. The combined filter is found to have better accuracy than its components in all test cases, and is only slightly slower than the EKF. Additionally, we show that this approach is robust to changing environmental conditions, has very low latency, and works even on low-cost processing hardware, which presents opportunities for low-cost mobile robot localization. In future work, this approach should also be tested in larger environments. 


\section{REFERENCES}

[1] H. Liu, H. Darabi, P. Banerjee, and J. Liu, "Survey of wireless indoor positioning techniques and systems," IEEE Transactions on Systems, Man and Cybernetics Part C: Applications and Reviews, vol. 37, no. 6, pp. 1067-1080, 2007.

[2] S. H. Fang and T. N. Lin, "Accurate WLAN indoor localization based on RSS fluctuations modeling," WISP 2009 - 6th IEEE International Symposium on Intelligent Signal Processing - Proceedings, pp. 27-30, 2009.

[3] Lighthouse, "Indoor Location Technologies Compared: GPS, WiFi, iBeacon \& RFID," https://lighthouse.io/ indoor-location-technologies-compared/, 2017, accessed: 2019-1022.

[4] S. Gezici, Z. Tian, G. B. Giannakis, H. Kobayashi, A. F. Molisch H. V. Poor, and Z. Sahinoglu, "Localization via ultra-wideband radios: A look at positioning aspects of future sensor networks," IEEE Signal Processing Magazine, vol. 22, no. 4, pp. 70-84, 2005.

[5] P. Dabove, V. Di Pietra, M. Piras, A. A. Jabbar, and S. A. Kazim, "Indoor positioning using Ultra-wide band (UWB) technologies: Positioning accuracies and sensors' performances," in 2018 IEEE/ION Position, Location and Navigation Symposium, PLANS 2018 - Proceedings. IEEE, apr 2018, pp. 175-184.

[6] H. Haas and C. Chen, "What is LiFi?" European Conference on Optical Communication, ECOC, vol. 2015-Novem, no. 6, pp. 1533-1544, 2015.

[7] S. De Lausnay, L. De Strycker, J. P. Goemaere, B. Nauwelaers, and N. Stevens, "A survey on multiple access Visible Light Positioning," 2016 IEEE International Conference on Emerging Technologies and Innovative Business Practices for the Transformation of Societies, EmergiTech 2016, pp. 38-42, 2016.

[8] T. H. Do and M. Yoo, "An in-depth survey of visible light communication based positioning systems," Sensors (Switzerland), vol. 16, no. 5, p. $678,2016$.

[9] C. Xie, W. Guan, Y. Wu, L. Fang, and Y. Cai, "The LED-ID Detection and Recognition Method Based on Visible Light Positioning Using Proximity Method," IEEE Photonics Journal, vol. 10, no. 2, pp. 1-16, 2018.

[10] R. Zhang, W. D. Zhong, Q. Kemao, and S. Zhang, "A Single LED Positioning System Based on Circle Projection," IEEE Photonics Journal, vol. 9, no. 4, 2017.

[11] J. Hao, J. Chen, and R. Wang, "Visible Light Positioning Using A Single LED Luminaire," IEEE Photonics Journal, vol. 11, no. 5, pp. 1-13, 2019.

[12] R. Mautz, "Indoor Positioning Technologies," Habilitation thesis, ETH Zurich, 2012. [Online]. Available: http://e-collection.library.ethz.ch/ eserv/eth:5659/eth-5659-01.pdf

[13] A. Sahin, Y. S. Eroglu, I. Guvenc, N. Pala, and M. Yuksel, "Hybrid 3-D Localization for Visible Light Communication Systems,' Journal of Lightwave Technology, vol. 33, no. 22, pp. 4589-4599, 2015.

[14] Q. Zou, W. Xia, Y. Zhu, J. Zhang, B. Huang, F. Yan, and L. Shen, "A VLC and IMU integration indoor positioning algorithm with weighted unscented Kalman filter," in 2017 3rd IEEE International Conference on Computer and Communications, ICCC 2017, vol. 2018-Janua, 2018, pp. 887-891.

[15] G. Simon, G. Zachar, and G. Vakulya, "Lookup: Robust and accurate indoor localization using visible light communication," IEEE Transactions on Instrumentation and Measurement, vol. 66, no. 9, pp. 2337-2348, 2017.

[16] Z. Li, L. Feng, and A. Yang, "Fusion based on visible light positioning and inertial navigation using extended kalman filters," Sensors (Switzerland), vol. 17, no. 5, p. 1093, may 2017.

[17] Z. Li, A. Yang, H. Lv, L. Feng, and W. Song, "Fusion of Visible Light Indoor Positioning and Inertial Navigation Based on Particle Filter," IEEE Photonics Journal, vol. 9, no. 5, 2017.

[18] C. Qin and X. Zhan, "VLIP: Tightly coupled visible-light/inertial positioning system to cope with intermittent outage," IEEE Photonics Technology Letters, vol. 31, no. 2, pp. 129-132, 2019.

[19] Y. Wu, W. Guan, X. Zhang, M. Huang, and J. Cao, "Visible light positioning system based on CMOS image sensor using particle filter tracking and detecting algorithm," Optics Communications, vol. 444, no. March, pp. 9-20, 2019.

[21] J. S. Gutmann, "Markov-Kalman localization for mobile robots," Proceedings - International Conference on Pattern Recognition, vol. 16, no. 2, pp. 601-604, 2002
[20] J. S. Gutmann and D. Fox, "An experimental comparison of localization methods continued," in IEEE International Conference on Intelligent Robots and Systems, vol. 1, no. 2, 2002, pp. 454-459.

[22] J. S. Gutmann and D. Fox, "An experimental comparison of localization methods continued," IEEE International Conference on Intelligent Robots and Systems, vol. 1, no. October, pp. 454-459, 2002.

[23] D. A. Zaugg, A. A. Samuel, D. E. Waagen, and H. A. Schmitt, "A combined particle/Kalman filter for improved tracking of beam aspect targets," IEEE Workshop on Statistical Signal Processing Proceedings, vol. 2003-Janua, pp. 535-538, 2003.

[24] J. N. Chi, L. H. Xie, P. Y. Zhang, Y. F. Lu, and G. S. Zhang, "Hybrid particle and Kalman filtering for pupil tracking in active IR illumination gaze tracking system," Mathematical Problems in Engineering, vol. 2014, 2014.

[25] P. J. Van Leeuwen, H. R. Künsch, L. Nerger, R. Potthast, and S. Reich, "Particle filters for high-dimensional geoscience applications: A review," Quarterly Journal of the Royal Meteorological Society, 2019.

[26] B. Béchadergue, W.-H. Shen, and H.-M. Tsai, "Comparison of ofdm and ook modulations for vehicle-to-vehicle visible light communication in real-world driving scenarios," Ad Hoc Networks, vol. 94, p. 101944, 2019.

[27] Yujin Robotics, "TurtleBot2," https://www.turtlebot.com/turtlebot2/, 2019, accessed: 2019-01-03. [Online]. Available: https://www.turtlebot. com/turtlebot $2 /$

[28] OpenMV, "OpenMV Cam H7 - OpenMV," https://openmv.io/products/ openmv-cam-h7, 2019, accessed: 2019-04-18. [Online]. Available: https://openmv.io/products/openmv-cam-h7

[29] J. M. Santos, D. Portugal, and R. P. Rocha, "An evaluation of 2D SLAM techniques available in Robot Operating System," 2013 IEEE International Symposium on Safety, Security, and Rescue Robotics, SSRR 2013, 2013.

[30] W. Hess, D. Kohler, H. Rapp, and D. Andor, "Real-time loop closure in 2D LIDAR SLAM," Proceedings - IEEE International Conference on Robotics and Automation, vol. 2016-June, pp. 1271-1278, 2016.

[31] M. Filipenko and I. Afanasyev, "Comparison of Various SLAM Systems for Mobile Robot in an Indoor Environment," 9th International Conference on Intelligent Systems 2018: Theory, Research and Innovation in Applications, IS 2018 - Proceedings, no. November, pp. 400-407, 2018.

[32] H. Jung, J.-h. Park, and H.-Y. Jeong, "Experimental Assessment of GNSS-based Vehicle Positioning Accuracy using 3-D SLAM," 2019 IEEE Vehicle Technology Conference, pp. 5-6, 2019.

[33] J. Hyun, T. Oh, H. Lim, and H. Myung, "UWB-based Indoor Localization Using Ray-tracing Algorithm," in 2019 16th International Conference on Ubiquitous Robots (UR). IEEE, 2019, pp. 98-101.

[34] S. Thrun, W. Burgard, and D. Fox, Probabilistic robotics. MIT press, 2005.

[35] Y. Bar-Shalom, X.-R. Li, and T. Kirubarajan, Estimation with Applications to Tracking and Navigation. John Wiley \& Sons, 2001.

[36] R. R. Labbe, Kalman and Bayesian Filters in Python, 2018, accessed: 2019-07-08. [Online]. Available: https://github.com/rlabbe/ Kalman-and-Bayesian-Filters-in-Python

[37] T. Li, M. Bolić, and P. M. Djurić, "Resampling Methods for Particle Filtering: Classification, implementation, and strategies," IEEE Signal Processing Magazine, vol. 32, no. 3, pp. 70-86, 2015

[38] R. Amsters, E. Demeester, P. Slaets, D. Holm, J. Joly, and N. Stevens, "Towards automated calibration of visible light positioning systems," in 2019 International Conference on Indoor Positioning and Indoor Navigation (IPIN), Sep. 2019, pp. 1-8

[39] Z. Zhang, "A flexible new technique for camera calibration," IEEE Transactions on Pattern Analysis and Machine Intelligence, vol. 22, no. 11 , pp. 1330-1334, 2000.

[40] J. Canny, "A Computational Approach to Edge Detection," IEEE Transactions on Pattern Analysis and Machine Intelligence, vol. PAMI-8, no. 6, pp. 679-698, 1986

[41] P. Sturm, Pinhole Camera Model. Boston, MA: Springer US, 2014, pp. 610-613.

[42] P. Stephan, I. Heck, P. Kraus, and G. Frey, "Evaluation of indoor positioning technologies under industrial application conditions in the SmartFactoryKL based on EN ISO 9283," IFAC Proceedings Volumes (IFAC-PapersOnline), vol. 13, no. PART 1, pp. 870-875, 2009.

[43] Alasdair, Allan, "The Raspberry Pi 3 Does Not Halt and Catch Fire - Make:," 2016. [Online]. Available: https://makezine.com/2016/03/ 02/raspberry-pi-3-not-halt-catch-fire/ 